\title{
Características de usuários de crack atendidos em um Centro de Atenção Psicossocial: concepção da equipe
}

\section{Health care workers' concept of characteristics of crack users treated at a psychosocial care center}

\author{
Annie Jeanninne Bisso Lacchini ${ }^{1}$, Cíntia Nasi ${ }^{2}$, Gustavo Costa de Oliveira ${ }^{3}$,
} Leandro Barbosa de Pinho ${ }^{4}$, Jacó Fernando Schneider ${ }^{5}$

\footnotetext{
${ }^{1}$ Enfermeira, Doutora em Enfermagem. Professor Adjunto do Departamento de Enfermagem da Universidade Federal de Ciências da Saúde de Porto Alegre (UFCSPA). Porto Alegre, RS, Brasil. E-mail: anniejbl@hotmail.com.

${ }^{2}$ Enfermeira, Doutora em Enfermagem. Professor Adjunto do Departamento de Enfermagem UFCSPA. Porto Alegre, RS, Brasil. E-mail: cintianasi@yahoo.com.br.

${ }^{3}$ Enfermeiro, Mestre em Enfermagem. Discente do Programa de Pós-Graduação em Enfermagem da Universidade Federal do Rio Grande do Sul (UFRGS), nível Doutorado. Porto Alegre, RS, Brasil. E-mail: gustavoenfufrgs@gmail.com.

${ }^{4}$ Enfermeiro, Doutor em Enfermagem Psiquiátrica. Professor Adjunto da Escola de Enfermagem (EE) da UFRGS. Porto Alegre, RS, Brasil. E-mail: Ibpinho@ufrgs.br.

${ }^{5}$ Enfermeiro, Doutor em Enfermagem. Professor Titular da EE/UFRGS. Porto Alegre, RS, Brasil. E-mail: jaco_schneider@uol.com.br.
}

\section{RESUMO}

O crack vem-se constituindo como um problema de saúde pública, face à sua natureza multifatorial, trazendo desafios aos trabalhadores. Este estudo objetivou conhecer a concepção de uma equipe de saúde mental sobre as características de usuários de crack atendidos em um Centro de Atenção Psicossocial (CAPS). Trata-se de um recorte de pesquisa avaliativa, qualitativa, do tipo estudo de caso, desenvolvida com oito profissionais da equipe de um CAPS. Segundo a equipe existem três características que definem os usuários de crack que frequentam o CAPS: 1) são usuários que demandam urgências de cuidados; 2) são usuários de difícil manejo e frágil adesão ao serviço e 3) são usuários que não possuem um perfil definido. Conclui-se que é preciso reconhecer as diferentes demandas trazidas aos serviços pelos usuários de crack, uma vez que exige uma re-organização dos serviços e a problematização das práticas para atender esta nova realidade.

Descritores: Saúde Mental; Serviços de Saúde; Transtornos Relacionados ao Uso de Substâncias; Enfermagem.

\section{ABSTRACT}

Crack use is a growing, multifactorial public health problem and presents challenges to health care workers. This study aimed to understand how a mental health team conceives of the characteristics of crack users treated in a psychosocial care center (CAPS) in the Brazilian public health care network. This is an evaluative, qualitative case study, developed with eight health care professionals who are members of a CAPS team. According to the team, there are three characteristics that define the crack users who receive care at the CAPS: 1 ) they require urgent care; 2 ) they are difficult to manage, and have low adherence to the service; and 3) they do not have a defined profile. The article concludes that it is necessary to recognize that crack users have different health care needs that require reorganization and questioning of practices in order to attend to this new reality.

Descriptors: Mental Health; Health Services; Substance-Related Disorders; Nursing. 


\section{INTRODUÇÃO}

No Brasil, o crack vem se tornando uma das drogas com maior repercussão social, constituindo-se em um grave problema de saúde pública. Estudos apontam que os usuários de crack constituem um grupo distinto entre os usuários de drogas, pois possuem características próprias, que necessitam de abordagem especial, devido ao processo acelerado de deterioração física e psíquica ${ }^{(1-}$ 3). Dados do II Levantamento Domiciliar sobre o uso de Drogas, realizado pelo CEBRID em 2005, constatou um aumento no consumo de $0,5 \%$ para $1,1 \%$. Nesse caso, o uso de crack tornou-se maior entre os homens em todas as faixas etárias, sendo que, entre homens de 12 a 24 anos, o uso foi de $2,9 \%^{(4)}$.

O Relatório Mundial sobre Drogas, de 2013, mostrou a extensão do problema associado com novas substâncias psicoativas e o impacto que elas podem ter sobre seus usuários. O texto aborda a questão sobre as drogas tradicionais, e que a ação internacional contra estas substâncias deve se concentrar tanto na oferta quanto na demanda. No entanto, problemas importantes como a falta de conhecimento, os efeitos adversos e riscos para a saúde pública e a segurança não parecem estar sob controle dessas ações internacionais ${ }^{(5)}$.

A produção científica acerca do consumo de crack tem se intensificado a partir da década de 1990, sendo objeto de estudo de vários autores nacionais e internacionais, especialmente do ponto de vista de evidenciar o perfil epidemiológico do usuário, o padrão de consumo e os efeitos fisiopatológicos da droga no $\operatorname{organismo}^{(6-8)}$. No entanto, a partir do paradigma da reforma psiquiátrica, que reconhece a relevância dos achados epidemiológicos, mas que, ao mesmo tempo, entende a pluralidade que envolve o uso prejudicado de drogas, entendemos que é preciso contextualizar a problemática e discuti-la à luz das novas tendências de cuidado, que envolvem os serviços comunitários, e do cotidiano das práticas dos profissionais de saúde.

No contexto brasileiro, desde 2010, tem-se constituído dentro da Política Nacional de Saúde Mental um debate mais preciso sobre o uso de drogas, trazendo novos desafios em relação à organização dos serviços e a necessidade de se repensar cotidianamente a prática dos trabalhadores. Isso quer dizer que, além de os Centros de Atenção Psicossocial (CAPS), como serviços substitutivos ao modelo do hospital especializado ${ }^{(9)}$ assumirem um papel estratégico no cuidado em saúde mental, é preciso investir numa rede que comporte os dispositivos comunitários no campo da saúde, as parcerias intersetoriais e os vários equipamentos sociais ${ }^{(10)}$.

Nesse sentido, embora tenhamos avançado de maneira substantiva no conhecimento sobre o perfil dos usuários e nas manifestações orgânicas da droga, entendemos que ainda carecem estudos que possam refletir sobre as repercussões no cotidiano do trabalho das equipes assistenciais. Vale lembrar que estamos longe de considerar o crack como uma droga que traz certo enquadramento de usuários a perfis específicos, mas conhecer essas características do usuário da droga a partir daquilo que trazem as equipes parece-nos uma importante contribuição para repensar e problematizar as atitudes dos profissionais.

Assim, neste estudo tivemos como objetivo conhecer a concepção de uma equipe de saúde mental sobre as características de usuários de crack atendidos em um Centro de Atenção Psicossocial (CAPS).

\section{METODOLOGIA}

O estudo é um recorte da pesquisa "Avaliação qualitativa da rede de serviços em saúde mental para atendimento a usuários de crack (ViaREDE)". Trata-se de uma pesquisa de natureza avaliativa, do tipo estudo de caso, que utilizou a Avaliação de Quarta Geração como referencial teórico-metodológico ${ }^{(11)}$, sendo financiada pelo CNPq/Ministério da Saúde (Edital MCT/CNPq 041/2010).

O cenário do estudo foi o Centro de Atenção Psicossocial para Álcool e outras Drogas (CAPS AD) do município de Viamão/RS. Trata-se de um CAPS AD II, que funciona das $08 \mathrm{~h}$ às $18 \mathrm{~h}$, nos dias da semana. Esse é o 
serviço de referência para atendimento a usuários de crack no município. Os sujeitos do estudo foram: oito trabalhadores do CAPS AD, 10 usuários do serviço, 11 familiares de usuários e sete gestores do sistema.

A coleta de dados da pesquisa ocorreu de janeiro a março de 2013, por meio de observações de campo e entrevistas. As observações de campo totalizaram 189 horas, sendo registradas em um diário de campo. Em relação às entrevistas, foram realizadas 36 ao todo. No entanto, os resultados deste artigo estão relacionados à aplicação das entrevistas aos oito profissionais do CAPS.

$\mathrm{Na}$ análise dos dados, as questões surgidas foram lidas, relidas e reagrupadas. Foram construídas, num primeiro momento, unidades de informação que, combinadas, deram origem a unidades de significado. Essas unidades reuniam núcleos centrais dos depoimentos que possuíam semelhança semântica, para, posteriormente, permitirem uma categorização. Com isso, surgiram três grandes eixos temáticos: organização da rede, organização do trabalho e relacionamento da sociedade com o usuário de crack. Dentro do último eixo, destacou-se a categoria temática "características do usuário de crack", a qual englobou três dimensões de discussão: a de que o usuário de crack demanda urgências do serviço, a de que o usuário é considerado pela equipe como um usuário difícil, tanto em relação ao manejo quanto à vinculação ao serviço, e de que o usuário de crack possui vários perfis.

A pesquisa foi submetida à avaliação pelo Comitê de Ética em Pesquisa da Universidade Federal do Rio Grande do Sul (UFRGS), recebendo parecer favorável à execução (protocolo 20157/2011). Também foi avaliada pelo CONEP/MS, sendo aprovada (parecer 337/2012).

Em relação aos aspectos éticos, seguimos o protocolo estabelecido pela Resolução 466/2012 do Conselho Nacional de Saúde, assim como a decisão de desistência por parte dos pesquisados, conforme Termo de Consentimento Livre e Esclarecido. Para isso, os integrantes da equipe foram identificados com a inicial
" $E$ ", seguido da ordem em que apareceram na entrevista. Exemplo: E6, E3.

\section{RESULTADOS E DISCUSSÃO}

Usuários de crack, em linhas gerais, constituem um grupo distinto entre os usuários de drogas. São usuários que se distinguem por possuírem características próprias, em razão da importante degradação física e psíquica causada pela substância. O usuário entra em um circuito que, com o uso continuado, vai levando a deterioração $^{(3,12)}$.

O próprio usuário de crack apresenta especificidades que nascem em razão de sua relação com esta droga, algo que é, em sua essência, resultado do mecanismo de ação da droga no organismo, como apontado em outros estudos $^{(13-14)}$. No entanto, é importante ressaltar que não queremos, na dimensão que estudamos (a das políticas de saúde), discorrer que o problema do usuário de crack está apenas na droga, e não em todo o contexto que o cerca. Estaríamos aqui pregando um reducionismo orgânico que não combina com as tendências contemporâneas do cuidado multifacetado do campo da saúde mental.

Nesse sentido, os trabalhadores afirmam que o uso da substância traz efeitos colaterais, bem característicos e manifestados organicamente. No entanto, esses efeitos também se traduzem na conduta desse usuário no interior dos serviços, trazendo novos desafios a eles.

Uma dessas características citadas pelos trabalhadores é de que o usuário de crack demanda "urgências", ou seja, são usuários que exigem cuidados imediatos para problemas que nem sempre podem ser resolvidos dessa maneira:

[...] a gente tem estas especificidades do crack, que são as que vêm vindo no decorrer, que é a rapidez com que eles pegam as coisas, a rapidez que é o próprio efeito da substância e que muitas vezes tem a ver com o jeito com que eles tratam o CAPS, que é um uso muito emergencial do serviço, "eu quero medicação, eu quero parar com a 
fissura", [...] tem a vibração do crack, da urgência do resolve a minha vida, do assim do: "eu quero que tu me dê alguma coisa porque a substância me dá tudo, o que que tu vai me dar que entre minimamente nesta competição sabe" "por que eu largaria isso?". E muitas vezes o trabalho é este de ver, bom o que você está perdendo, por que tu ta aqui? (E1)

É eu acho que o usuário de crack ele é um usuário com o perfil imediatista, ele quer tudo na hora, aqui e agora, às vezes eles esperam acumular as dificuldades, os problemas, que às vezes poderiam ser trabalhados num momento em que a pessoa ta numa situação mais tranquila, mas não, daí acabam esperando até não aguentar mais, ai eles querem vir pra cá e resolverem tudo naquele dia. Eles acabam não tendo muita tolerância para aquele tempo de espera, aí querem ter noção imediata, querem medicação imediata, querem tudo muito imediato e eu acho que isso tem a ver com a questão um pouco como funciona a droga no organismo daquele sujeito (E2)

Cada droga tem seu mecanismo de ação particular. Em especial ao crack, é uma droga que possui um sistema de ação muito rápido e o padrão de consumo intenso, contínuo, associado a ciclos intermitentes de parada abrupta, trazem ao indivíduo grande mal estar físico e psicológico. Nessa urgência pelo crack que a pessoa vai se expondo a situações de risco, e na medida em que procuram sair do consumo compulsivo, recaem novamente no circuito ${ }^{(15)}$.

Apesar das particularidades de cada substância, a grande maioria das drogas age direta e indiretamente no mesmo local no cérebro, provocando quadros clínicos peculiares. Por isso, é relevante que a definição do diagnóstico seja aplicada de maneira correta, pois permite estabelecer um plano terapêutico apropriado, com intervenções específicas para cada paciente ${ }^{(16-17)}$. Nesse sentido, conhecer a especificidade da substância pode aumentar não somente o envolvimento do trabalhador com o usuário, como também sua participação nas atividades oferecidas dentro e fora dos serviços de saúde.

Há dois aspectos considerados importantes no manejo a usuários de crack, que são a sua imprevisibilidade e inscrição da droga na história desse usuário $^{(18)}$. Por mais que não seja a intenção a padronização de cada usuário, é importante reconhecer que a droga traz efeitos imediatos e comportamentos compulsivos que refletem em estratégias inapropriadas quando em tratamento. Isso tudo revela a grande relação que o usuário estabelece com droga, sendo necessário conhecê-la primeiro, para depois poder pensar em possibilidades de intervenção:

\section{[...] não tem um atendimento não tem um pronto} atendimento para essa população e eu acho que esse cuidado imediatista que eles querem é a própria relação com droga né, de querer mais, da fissura, da agitação daquela coisa de querer mais, mais, mais... (E3)

O profissional mostra a necessidade de haver um pronto-atendimento para essa população, ao despeito de uma orientação normativa que nos designa os CAPS AD como serviços especializados nesse atendimento. Parece haver em seu depoimento uma preocupação de olhar $a$ priori para o relacionamento dessa substância com o sujeito, tornando mais amplo seu papel na intervenção, pois além de permear o conhecimento da fissura e das repercussões orgânicas dela, esse comportamento também se reflete no uso emergencial que fazem do serviço, questão essa já apontada em outro estudo ${ }^{(19)}$. Um ponto importante, ao nosso ver, para ser problematizado pari passu no cotidiano do processo de trabalho do CAPS e dos serviços da rede de atenção psicossocial.

Nesse sentido, entendemos que cada serviço de saúde precisa poder trabalhar num modelo de organização que atenda a demanda do usuário, reconheça a influência dos efeitos da droga no organismo e os comportamentos associados a ele. Desse modo, o ato de cuidar dos usuários de crack em serviços comunitários 
parece requerer certo conhecimento criativo e reflexivo, uma vez que é preciso aprender com o imediatismo, ao tempo em que se tenta ressignificar a relação estabelecida com a droga.

Esse uso emergencial que o usuário faz do CAPS gera uma vinculação precária com o serviço. Para o trabalhador, o usuário de crack é um usuário "difícil", que responde menos ao manejo terapêutico e adere pouco às atividades e rotinas assistenciais:

\section{[...] o usuário de crack geralmente é bem difícil, é bem raro} ele ser usuário só de crack, geralmente ele de fato já passou por outras substâncias até pelo histórico da substância mesmo[...] (E1)

O usuário de crack se vincula menos ao serviço: "ele vai e volta".(E3)

A partir das características imediatistas do usuário, não deve caber apenas ao CAPS AD o ônus de suportar o abandono e o vínculo precário com o serviço. Por mais que os usuários de crack sejam mais propícios a abandonarem o tratamento $^{(3)}$, o trabalho em rede é fundamental para garantir certa continuidade no acompanhamento. Assim, é necessário investir em parceria com outros setores públicos e equipamentos sociais, no sentido de proporcionar busca ativa de casos, gerenciamento compartilhado do cuidado e corresponsabilização das equipes de saúde ${ }^{(20)}$.

Além disso, consideramos a relevância de se pensar que o funcionamento irregular e imediatista do usuário deve ser pauta constante das reuniões de equipe, uma vez que o consumo prejudicado gera determinados descompassos que são sentidos pelo trabalhador. Descompassos esses que revelam o quão intensa é a relação da droga com o sujeito, e a ressignificação dessa relação é um dos grandes desafios no campo.

No que diz respeito ainda à fragilidade dos vínculos do usuário com o serviço, outro ponto destacado pelos trabalhadores diz respeito às divergências existentes entre o horário de funcionamento do CAPS e o horário de funcionamento do usuário. Enquanto o CAPS possui uma rotina diurna de trabalho, o usuário tem uma vida noturna, fazendo com que ele use o dia para se recuperar dos efeitos da substância:

E o horário também, geralmente esses usuários nos momentos mais críticos da vida dele a noite, de manhã o cara não vem o cara tá dormindo. Tu vai visitar o cara de manhã, tu não vai achar ninguém em casa né, ou ele vai estar dormindo ou ele não vai te atender... (E3)

O usuário de crack ele não se encaixa assim, por exemplo, vamos sentar e fazer uma atividade lúdica, ele não quer. É muito difícil tu sentar com alguém e dizer - tá agora vamos fazer uma dobradura, ou uma pintura. Realmente, eu não vejo eles conseguir sentar, eles querem ficar sentados vendo televisão [...] (E5)

Dentre os desafios de constituir um sistema de saúde mais adaptado às necessidades das pessoas, considera-se que o usuário do serviço seria o elemento estruturante de todo o processo de produção de saúde, de modo a romper com a clínica tradicional que insiste em enxergálo em parcelas. O desafio dos serviços seria o de construir linhas de cuidado a partir dos desejos, ou seja, daquilo que o sujeito entende como problema em sua vida, sem desconsiderar sua história e suas subjetividades ${ }^{(21)}$.

Nesse sentido, de acordo com os paradigmas reformadores, parece haver uma necessidade de conhecer melhor a população usuária de uma substância como o crack, além de perceber que, a despeito de funcionamentos muito parecidos, cada sujeito tem uma situação emergencial singular. Isso traz o desafio ao CAPS de planejar ações sem se descontextualizar das culturas, das relações, da circulação do usuário pelos espaços, enfim, das experiências que movem o sujeito, pois são essas características, singulares por natureza, que devem orientar a prática estabelecida pelos trabalhadores dos serviços $^{(22)}$.

A necessidade de repensar a organização dos serviços de saúde mental é urgente, diante da conjuntura 
complexa que envolve o sujeito, suas relações e a droga. No caso do crack, formar vínculos mais genuínos e duradouros com o usuário predispõe a urgência de se problematizar o funcionamento do CAPS. Possíveis saídas para isso seria a parceria com equipes de Consultório na Rua, o deslocamento de equipes itinerantes que possam circular pelos espaços e cenários das pessoas em horários longe dos habituais. Consideramos que o CAPS enriqueceria seu cardápio de atuação na medida em que passe a atuar fora do espaço extramuros e também longe de organizações mais tradicionais dos serviços, potencializando vínculos.

Diante disso, é possível entender que esses usuários se inscrevem no mundo da droga e na sociedade de outras maneiras, trazem desafios ao cotidiano dos serviços não somente pela fraca adesão, mas também pelo manejo complexo e multidimensional que é o uso prejudicado de drogas. Agindo dessa maneira, o CAPS inicia o processo de construção de uma linha de cuidado que valorize as diferenças e os diferentes.

Além das questões que envolvem a urgência e os vínculos precários, o trabalhador destacada que o perfil do usuário atendido pelo CAPS AD é muito variado, diferente daquela imagem proposta pela sociedade de um usuário degradado, marginalizado e esquecido:

\section{[...] E o perfil destas pessoas é muito variado, tem gente} que é caminhoneiro e que ta aí nas estradas, mas é usuário de crack, tem gente que vive pro crack, mas este perfil midiático, que não é o preponderante, tem várias pessoas que seguem a sua vida e que tem um trabalho, claro que vem os efeitos disso... (E1)

Eu não vejo um perfil definido porque aqui a gente pega às vezes gurizinhos de 19 anos que a recém saiu da adolescência e às vezes pegamos pai de família de 40 e poucos anos e às vezes tu pega o que rouba e o que trabalha consegue manter o seu trabalho para manter $o$ vício. Eu acho complicado um perfil, eu acho que não tem um perfil é muito variável [...](E4)
No contexto de uso de drogas, faz-se necessário reconhecer o consumidor, suas características e necessidades, e buscar novas estratégias de contato e de vínculo com ele e sua família. O desenho de abordagens capazes de chegar às necessidades individuais perpassa também o conhecimento sobre a amplitude desses perfis, nem sempre relacionado àquilo que nossa sociedade enxerga ou defende ${ }^{(23)}$ :

Às vezes a gente pega e já vê com todo aquele perfil emagrecido, sem forças e ás vezes não, às vezes tu pega um que se tu visse na rua tu nunca iria sonhar esse ai usuário de qualquer coisa, tu nunca iria imaginar. (E4) O usuário de crack quando chega ao serviço, na minoria das vezes ele, estava com aquela aparência horrorosa que aparece na TV, não vou mentir que a pessoa vai chegar com a aparência emagrecida, sem força, outras patologias envolvendo devido ao uso, mas o que acontece realmente são aqueles usuário que usam fim de semana, trabalham pra pagar o seu uso, e eu vou dizer que a maioria dos usuários que vêm ao CAPS são assim... os que roubam e ficam com dívidas, são as situações mais complicadas, porque esses vem ao CAPS por causa do desespero dos familiares, e no desespero dos familiares que já foram pra vida de marginal é internar e sumi com a pessoa pra resolver com problemas que, muitas vezes, não são problemas de saúde, são problemas sociais que muitas vezes pessoais que não dizem respeito a saúde. (E6)

No entanto, o depoimento de E8 nos destaca que o usuário vivenciado pela equipe do CAPS muitas vezes é aquele que está confinado sob seus muros, ou seja, um "público estratificado", bem diferente do mundo extramuros:

Quando ocorreu a coisa, tem relação com outras coisas que eu já comentei que foi a respeito do acesso do perfil de usuários, que talvez a gente não tenha esse perfil da mídia, eu tento me distanciar, porque a gente tem uma 
amostra viciada... do público, eu digo viciada porque... é bom de falar (risos) a gente tem um público mais reciclado aqui sabe (risos) e aí, é nós temos um público mais selecionado aqui, mais estratificado, melhor e talvez a gente não esteja vendo esse pessoal mais grave, assim. A gente vê quando chegam do Ministério Público, a gente já presenciou situações deploráveis assim, então talvez seja uma dificuldade de acesso, no caso a gente não vê esse usuário propagado pela mídia, porque existir, existe, óbvio que tem um monte de fantasias, mas aqueles extremos da ponta existem, tem usuário que perde tudo aqui... (E8)

Diante das falas, observamos que em torno da droga, nesse caso o crack, se estruturam e se disseminam discursos que consolidam uma ideia simplista a respeito do perfil do usuário de crack. Essa simplificação deprecia um contexto que se mostra complexo, implicando em danos ao indivíduo e sociedade, uma vez que se instituem rótulos que podem gerar preconceitos e exclusão desse usuário do meio social.

Ao se considerar um contexto social amplo, nota-se que o crack se configura como símbolo do consumo das camadas mais populares; a droga acessível às camadas mais desfavorecidas economicamente. No entanto, simboliza, ao mesmo tempo, a degradação, a mercadoria originada de um trato refinado da cocaína e associada às camadas empresariais, símbolo de ostentação, poder e excitação intelectual $^{(24)}$.

Desse modo, percebemos o usuário de crack em diversos cenários, correlacionando o uso da droga a diversos fatores socioeconômicos, psicológicos e culturais. Produto da busca obstinada por efeitos positivos, o uso de crack perpassa a realização de atividades ilícitas na falta de recursos econômicos à aquisição da droga, visto que o consumo do crack abarca relações sociais em contexto de centralidades e periferias, evidenciando a complexidade a respeito do perfil do usuário dessa droga ${ }^{(25)}$.
Além disso, frente ao uso do crack tem se instituído ações em saúde que fortalecem discursos ideológicos, os quais o demonstram como uma adversidade social responsável pela degradação da sociedade. Consolidado como uma problemática social, o crack, como também os usuários recebem atenção da mídia, ocupando, nitidamente, espaço em reportagens policiais em que há correlação com a violência social, sendo sugerida, em inúmeras ocasiões, a "limpeza na sociedade" para que haja o restabelecimento da ordem.

Diariamente, acompanham-se na mídia televisiva e impressa casos de filhos que são acorrentados em casa pelos próprios pais para não usarem drogas ou roubarem objetos para comprar drogas, mas sabemos que as perdas não são apenas materiais. Frequentemente, os usuários de crack apresentam dificuldades em verbalizar sobre si para outra pessoa, pois sentem medo de serem discriminados pelos seus atos, evitando, com isso, a busca por atendimento em saúde. E esse é apenas uma das "pontas do iceberg", vividas pela família e pela comunidade, e que precisa ser alcançada pela CAPS. Isso nos retrata o quanto é necessário que o CAPS conheça mais a realidade das pessoas.

Nesse contexto, entendemos que as características do usuário de crack são relevantes para que os serviços possam adquirir novos e diferentes contornos, tanto pela forma de cuidar do usuário de crack, como também pela relação que ele ocupa na organização do trabalho dos serviços da rede.

\section{CONSIDERAÇÕES FINAIS}

A partir do cenário de pano de fundo, este estudo permitiu conhecer a concepção de uma equipe de um CAPS AD II quanto às características de usuários de crack atendidos nesse serviço de saúde. Fica notório que, entre as concepções desses trabalhadores, encontram-se três principais características que definem os usuários:

1) usuários que demandam urgências de cuidados;

2) usuários de difícil manejo e frágil adesão ao serviço e; 
3) usuários que não possuem perfil definido.

Ainda, o presente estudo revelou que as práticas asilares em saúde mental não constituíram os relatos dos profissionais da equipe acercar das concepções, angariando ações em saúde mental direcionadas ao modo de Atenção Psicossocial. Com isso, essas concepções direcionam-se à caracterização de práticas focadas no usuário, valorizando-se seu contexto social e permitindo sua participação ativa ao longo da terapêutica em saúde mental.

A partir disso, salientamos que essas concepções, estando no plano das falas de profissionais de saúde mental, evidenciam o impacto desse estudo, uma vez que podem indicar um avanço na consolidação da reforma psiquiátrica brasileira, na medida em que há sensibilização da equipe e execução de ações que

\section{REFERÊNCIAS}

1. Factor SH, Sackoff JE, Raj-Singh S, Wu Y, Monserrate J, Munsiff S, et al. Street-outreach Improves Detection but not Referral for Drug Users with Latent Tuberculosis, New York City. Subst Use Misuse [Internet]. 2011 [acesso em: 30 jun de 2015];46(14):1711-5. Disponível em: http://dx.doi.org/10.3109/10826084.2011.615562. 2. Guindalini C, Vallada H, Breen G, Laranjeira R. Concurrent crack and powder cocaine users from Sao Paulo: Do they represent a different group? BMC Public Health [Internet]. 2006 [acesso em: 30 jun de 2015];6:10. Disponível em:

http://dx.doi.org/10.1186/1471-2458-6-10.

3. Duailibi LB, Ribeiro M, Laranjeira R. Profile of cocaine and crack users in Brazil. Cad Saude Publica [Internet]. 2008 [acesso em: 30 jun de 2015];24 (Suppl 4):s545-57. Disponível em:

http://dx.doi.org/10.1590/\$0102-311X2008001600007.

4. Laranjeira R. II Levantamento Nacional de álcool e drogas: o uso de cocaína e crack no Brasil. São Paulo: Unifesp, Inpad/Uniad; 2012.

5. United Nations. World Drug Report. Geneva (Switzerland): United Nations; 2013. 148 p.

6. Meyer VJ, Little DM, Fitzgerald DA, Sundermann EE, Rubin LH, Martin EM, et al. Crack cocaine use impairs anterior cingulate and prefrontal cortex function in women with HIV infection. J

Neurovirol [Internet]. 2014 [acesso em: 30 jun de 2015];20(4):35261. Disponível em: http://dx.doi.org/10.1007/s13365-014-0250-x. 7. Mann CG, Bastos FI. A Brief Photo-biography of Crack Users From Rio de Janeiro's Metropolitan Area: Their Settings and Tools. Subst Use Misuse [Internet]. 2015 [acesso em: 30 jun de 2015];50(4):520-2. Recuperado de:

http://dx.doi.org/10.3109/10826084.2015.978198.

8. Sterk CE, Elifson KW, DePadilla L. Neighbourhood structural characteristics and crack cocaine use: exploring the impact of perceived neighbourhood disorder on use among African imprimem novas realidades e modelagens assistenciais. Entretanto, há necessidade de novas pesquisas que abordem o planejamento e resultados dessas ações, construtos que exprimem uma importante limitação dessa pesquisa.

Por fim, sendo o crack um problema de saúde pública, torna-se importante considerar o uso do crack como um relevante e frutífero campo para novas investigações na área da saúde mental, no sentido de evidenciar que, no contexto da atenção psicossocial e da reforma psiquiátrica, é preciso cada vez mais problematizar os modos de operar dos serviços, as formas de organização, como também incorporar as expectativas/experiências dos trabalhadores e dos usuários ao cuidado no cotidiano dos serviços de saúde.

Americans. Int J Drug Policy [Internet]. 2014 [acesso em: 30 jun de 2015];25(3):616-23. Disponível em:

9. Miranda L, Campos RTO. Análise do trabalho de referência em Centros de Atenção Psicossocial. Rev Saude Publica [Internet]. 2008 [acesso em: 30 jun de 2015];42(5):907-13. Disponível em: http://dx.doi.org/10.1590/S0034-89102008005000051.

10. Guimarães JMX, Jorge MSB, Assis MMA. (In)satisfação com o trabalho em saúde mental: um estudo em Centros de Atenção Psicossocial. Cien Saude Colet [Internet]. 2011 [acesso em: 30 jun 2015];16(4):2145-54. Disponível em:

http://dx.doi.org/10.1590/S1413-81232011000400014.

11. Guba EG, Lincoln YS. Avaliação de Quarta Geração. Campinas (SP): UNICAMP; 2011.

12. Cunha PJ, Bechara A, de Andrade AG, Nicastri S. Decisionmaking deficits linked to real-life social dysfunction in crack cocaine-dependent individuals. Am J Addict. 2011 [acesso em: 30 jun 2015];20(1):78-86. Disponível em: http://dx.doi.org/10.1111/j.1521-0391.2010.00097.x.

13. Dickson-Gomez J, Bodnar G, Guevara CE, Rodriguez K, De Mendoza LR, Corbett AM. With God's Help I Can Do It: Crack Users' Formal and Informal Recovery Experiences in El Salvador. Subst Use Misuse [Internet]. 2011 [acesso em: 30 jun 2015];46(4):42639. Disponível em:

http://dx.doi.org/10.3109/10826084.2010.495762.

14. Smith MJ, Thirthalli J, Abdallah AB, Murray RM, Cottler LB. Prevalence of psychotic symptoms in substance users: a comparison across substances. Compr Psychiatry [Internet]. 2009 [acesso em: 30 jun 2015];50(3):245-50. Disponível em: http://dx.doi.org/10.1016/j.comppsych.2008.07.009. 15. Chaves T V, Sanchez ZM, Ribeiro LA, Nappo SA. Fissura por crack: comportamentos e estratégias de controle de usuários e exusuários. Rev Saude Publica [Internet]. 2011 [acesso em: 30 jun 
2015];45(6):1168-75. Disponível em:

http://dx.doi.org/10.1590/S0034-89102011005000066.

16. Silva LHP, Borba LO, Paes MR, Guimarães AN, Mantovani MF, Maftum MA. Perfil dos dependentes químicos atendidos em uma unidade de reabilitação de um hospital psiquiátrico. Esc Anna Nery [Internet]. 2010 [acesso em: 30 jun 2015];14(3):585-90. Disponível em: http://dx.doi.org/10.1590/S1414-81452010000300021.

17. Guimarães CF, Santos DVV, Freitas RC, Araujo RB. Perfil do usuário de crack e fatores relacionados à criminalidade em unidade de internação para desintoxicação no Hospital Psiquiátrico São Pedro de Porto Alegre (RS). Rev Psiquiatr do Rio Gd do Sul [Internet]. 2008 [acesso em: 30 jun 2015];30(2). Disponível em: http://dx.doi.org/10.1590/S0101-81082008000300005.

18. Lima M, Jucá VJS, Nunes MO, Ottoni VE. Signos, significados e práticas de manejo da crise em Centros de Atenção Psicossocial. Interface - Comun Saúde, Educ [Internet]. 2012 [acesso em: 30 jun $\left.2^{\circ} 15\right]$;16(41):423-34. Disponível em:

http://dx.doi.org/10.1590/S1414-32832012000200011.

19. Gabatz RIB, Schmidt AL, Terra MG, Padoin SM de M, Silva AA da, Lacchini AJB. Perception of crack users in relation to use and treatment. Rev Gaúcha Enferm. 2013 [acesso em: 30 jun 2015];34(1):140-6. Disponível em:

http://dx.doi.org/10.1590/S1983-14472013000100018.

20. Xavier RT, Monteiro JK. Tratamento de Pacientes Usuários de crack e outras drogas nos CAPS AD. Psicologia Revista [Internet]. 2013 [acesso em: 30 jun 2015];22(1):61-82. Disponível em: http://revistas.pucsp.br/index.php/psicorevista/article/view/1665 8.

21. Franco TB, Merhy EE. A produção imaginária da demanda e o processo de trabalho em saúde. In: Pinheiro R, Matos RA.

Construção social da demanda: direito à saúde, trabalho em equipe, participação e espaços públicos. Rio de Janeiro: CEPESC/UERJ/ABRASCO; 2005. p. 181-93.

22. Horta RL, Horta BL, Rosset AP, Horta CL. Perfil dos usuários de crack que buscam atendimento em Centros de Atenção Psicossocial. Cad Saude Publica [Internet]. 2011 [acesso em: 30 jun 2015];27(11):2263-70. Disponível em:

http://dx.doi.org/10.1590/\$0102-311X2011001100019.

23.

23. Gabinete de Segurança Institucional; Conselho Nacional Antidrogas. Resolução № 03/GSIPR/CH/CONAD, de 27 de outubro de 2005. Aprova a Política Nacional Sobre Drogas. Conselho Nacional Antidrogas. Brasília: Conselho Nacional Antidrogas; 2005. 24. Jorge MSB, Quinderé PHD, Yasui S, Albuquerque RA. Ritual de consumo do crack: aspectos socioantropológicos e repercussões para a saúde dos usuários. Cien Saude Colet [Internet]. 2013 [acesso em: 30 jun 2015];18(10):2909-18. Disponível em: http://dx.doi.org/10.1590/S1413-81232013001000015.

25. Ali H, Bushra R, Aslam N. Profile of drug users in Karachi City, Pakistan. East Mediterr Health J [Internet]. 2011 [acesso em: 30 jun 2015];17(1):41-5. Disponível em:

http://applications.emro.who.int/emhj/V17/01/17_1_2011_0041_ 0045.pdf?ua=1.

Recebido: 27/03/2014.

Aceito: 02/07/2014.

Publicado: 30/06/2015. 